hep-th/0210043

\title{
Poisson geometry of sigma models with extended supersymmetry
}

\author{
Simon Lyakhovich ${ }^{a 1}$ and Maxim Zabzine ${ }^{b 2}$ \\ ${ }^{a}$ Department of Theoretical Physics, Tomsk State University, \\ 634050, Lenin av. 36, Tomsk, Russia \\ ${ }^{b}$ INFN Sezione di Firenze, Dipartimento di Fisica, \\ Via Sansone 1, I-50019 Sesto F.no (FI), Italy
}

\begin{abstract}
We consider a general $\mathrm{N}=(2,2)$ non-linear sigma model with a torsion. We show that the consistency of $\mathrm{N}=(2,2)$ supersymmetry implies that the target manifold is necessary equipped with two (in general, different) Poisson structures. Finally we argue that the Poisson geometry of the target space is a characteristic feature of the sigma models with extended supersymmetry.
\end{abstract}

\footnotetext{
${ }^{1}$ e-mail address: sll@phys.tsu.ru

${ }^{2}$ e-mail address: zabzine@fi.infn.it
} 


\section{Introduction}

The supersymmetric non-linear sigma models with extended supersymmetry play a prominent role both in physics and mathematics. From the physical point of view these models are of manifest interest to the string theory. On the mathematical side there is an intriguing relation between these models and the complex geometry which has been well recognized already in eighties [1]-[5].

In the present paper we would like to take a fresh look at the geometry of the general $\mathrm{N}=(2,2)$ supersymmetric two dimensional non-linear sigma model. Our study reveals a deep relationship between Poisson geometry and supersymmetry. In particular we show that the consistency of $\mathrm{N}=(2,2)$ supersymmetry implies that the target manifold is necessary equipped with two (in general, different) Poisson structures.

In a certain sense this situation is similar to the case of topological Poisson sigma models $[6,7]$ where the consistency of gauge symmetry requires the target manifold to have a Poisson structure. These models have been efficiently exploited for the deformation quantization and have brought a natural generating procedure [8] for the Kontsevich star product [9]. The method of topological Poisson sigma models combined with the Fedosov approach [11] allows to construct explicitly the covariant star-products [10].

Starting from Zumino's observation [1] that the target manifold of the $\mathrm{N}=2$ supersymmetric sigma model needs to be Kähler, it has been recognized that supersymmetry and complex geometry go hand in hand. In [2], Alvarez-Gaumé and Freedman classified possible supersymmetric sigma models in two dimensions. Later in [3] it was remarked that more general supersymmetric sigma model can be constructed by including Wess-Zumino term in the action. Gates, Hull and Roček described the general N=2 supersymmetric sigma model with torsion [4]. The target manifold of such model should be a bihermitian complex manifold with extra non-trivial constraints on the complex structures. Ever since the work of Gates-Hull-Roček these models have attracted a lot of attention, e.g. [14]-[19] (also the $\mathrm{N}=2 \mathrm{WZW}$ models provide important examples of this geometry, [20]-[22]). However the nature of those non-trivial constraints on the complex structure remains unclear. In this paper we reformulate and interpret these non-trivial constraints on the complex structures.

More specifically, we study these constraints on the background geometry not in the terms of complex structures and exterior forms, but in the terms of the antisymmetric contravariant tensor analysis (using the Schouten brackets). As a result we observe that the consistency conditions of the $\mathrm{N}=(2,2)$ supersymmetry for the model require a bi-Poisson structure to exist on the target manifold.

This paper is organized as follows. In Section 2 we review the results from the Gates- 
Hull-Roček work [4] on the $\mathrm{N}=2$ supersymmetric sigma model with torsion. Following this introductory section in Section 3 we proceed to the reformulation of the geometry in terms of contravariant antisymmetric tensors and the Schouten bracket. Due to this reformulation of the geometric conditions we arrive at the claim that the bihermitian complex manifold which supports the $\mathrm{N}=(2,2)$ model is bi-Poisson (i.e., there exist two Poisson structures). In next Section 4 using the fact of the existence of two Poisson structures we explore the geometry of the manifolds and propose a classification program for them. In Section 5 we look at the generalizations of the presented logic for the $\mathrm{N}=4$ supersymmetric sigma model. Finally in Section 6 we give a summary of the paper and discuss the open problems. We argue that the appearance of the Poisson structures is a common feature of the sigma models with extended supersymmetry.

In this paper we attempt to clarify the relation of supersymmetry and Poisson geometry in terms accessible to both mathematicians and physicists. To this end, we review some basic and well known notions in terms intended to make them accessible to a new audience.

\section{$2 \mathrm{~N}=(2,2)$ supersymmetric sigma model}

In this section we review the results on the general $\mathrm{N}=(2,2)$ supersymmetric sigma model from the original work [4]. This will allow us to introduce the notation and some relevant concepts.

Let us start from the general $\mathrm{N}=1$ sigma model which is written in $\mathrm{N}=1$ superfields (see Appendix for the conventions)

$$
S=\int d^{2} \sigma d^{2} \theta D_{+} \Phi^{\mu} D_{-} \Phi^{\nu}\left(g_{\mu \nu}(\Phi)+B_{\mu \nu}(\Phi)\right)
$$

The action (2.1) is manifestly supersymmetric under the supersymmetry transformations

$$
\delta^{1}(\epsilon) \Phi^{\mu}=i\left(\epsilon^{+} Q_{+}+\epsilon^{-} Q_{-}\right) \Phi^{\mu}
$$

which form the standard supersymmetry algebra

$$
\left[\delta^{1}\left(\epsilon_{1}\right), \delta^{1}\left(\epsilon_{2}\right)\right] \Phi^{\mu}=2 i \epsilon_{1}^{+} \epsilon_{2}^{+} \partial_{+} \Phi^{\mu}+2 i \epsilon_{1}^{-} \epsilon_{2}^{-} \partial_{=} \Phi^{\mu}
$$

We look for additional supersymmetry transformations of the form

$$
\begin{gathered}
\delta^{2}(\epsilon) \Phi^{\mu}=\epsilon^{\alpha} D_{\alpha} \Phi^{\nu} L_{\nu}^{\mu}(\Phi)+\epsilon^{\alpha}\left(\gamma_{3} D\right)_{\alpha} \Phi^{\nu} M_{\nu}^{\mu}(\Phi)= \\
=\epsilon^{+} D_{+} \Phi^{\nu} J_{+\nu}^{\mu}(\Phi)+\epsilon^{-} D_{-} \Phi^{\nu} J_{-\nu}^{\mu}(\Phi)
\end{gathered}
$$


where

$$
L_{\nu}^{\mu}=\frac{1}{2}\left(J_{+\nu}^{\mu}+J_{-\nu}^{\mu}\right), \quad M_{\nu}^{\mu}=\frac{1}{2}\left(J_{+\nu}^{\mu}-J_{-\nu}^{\mu}\right) .
$$

Classically the ansatz (2.4) is unique for dimensional reasons. It turns out that the action (2.1) is invariant under the transformations (2.4) provided that

$$
J_{ \pm \rho}^{\mu} g_{\mu \nu}=-g_{\mu \rho} J_{ \pm \nu}^{\mu}
$$

and

$$
\nabla_{\rho}^{( \pm)} J_{ \pm \nu}^{\mu} \equiv J_{ \pm \nu, \rho}^{\mu}+\Gamma_{\rho \sigma}^{ \pm \mu} J_{ \pm \nu}^{\sigma}-\Gamma_{\rho \nu}^{ \pm \sigma} J_{ \pm \sigma}^{\mu}=0
$$

where one defines two affine connections

$$
\Gamma_{\rho \nu}^{ \pm \mu}=\Gamma_{\rho \nu}^{\mu} \pm g^{\mu \sigma} H_{\sigma \rho \nu}
$$

with $H$ being the torsion three form,

$$
H_{\mu \rho \sigma}=\frac{1}{2}\left(B_{\mu \rho, \sigma}+B_{\rho \sigma, \mu}+B_{\sigma \mu, \rho}\right) .
$$

Next we have to require the standard on-shell $\mathrm{N}=2$ supersymmetry algebra, i.e. the first supersymmetry transformations (2.2) and the second supersymmetry transformations (2.4) commute

$$
\left[\delta^{2}\left(\epsilon_{1}\right), \delta^{1}\left(\epsilon_{2}\right)\right] \Phi^{\mu}=0
$$

and the commutator of two second supersymmetry transformations

$$
\begin{gathered}
{\left[\delta^{2}\left(\epsilon_{1}\right), \delta^{2}\left(\epsilon_{2}\right)\right] \Phi^{\mu}=-2 i \epsilon_{1}^{+} \epsilon_{2}^{+} \partial_{+} \Phi^{\lambda}\left(J_{+\lambda}^{\nu} J_{+\nu}^{\mu}\right)-2 i \epsilon_{1}^{-} \epsilon_{2}^{-} \partial_{=} \Phi^{\lambda}\left(J_{-\lambda}^{\nu} J_{-\nu}^{\mu}\right)+} \\
+\epsilon_{1}^{+} \epsilon_{2}^{+} D_{+} \Phi^{\lambda} D_{+} \Phi^{\rho} \mathcal{N}_{\lambda \rho}^{\mu}\left(J_{+}\right)+\epsilon_{1}^{-} \epsilon_{2}^{-} D_{-} \Phi^{\lambda} D_{-} \Phi^{\rho} \mathcal{N}_{\lambda \rho}^{\mu}\left(J_{-}\right)
\end{gathered}
$$

should satisfy the same algebra as the first (2.3), i.e.

$$
\left[\delta^{2}\left(\epsilon_{1}\right), \delta^{2}\left(\epsilon_{2}\right)\right] \Phi^{\mu}=2 i \epsilon_{1}^{+} \epsilon_{2}^{+} \partial_{+} \Phi^{\mu}+2 i \epsilon_{1}^{-} \epsilon_{2}^{-} \partial_{=} \Phi^{\mu} .
$$

In $(2.11) \mathcal{N}$ is the Nijenhuis tensor which is defined as follows

$$
\mathcal{N}_{\mu \nu}^{\rho}\left(J_{ \pm}\right)=J_{ \pm \mu}^{\gamma} \partial_{[\gamma} J_{ \pm \nu]}^{\rho}-J_{ \pm \nu}^{\gamma} \partial_{[\gamma} J_{ \pm \mu]}^{\rho} .
$$

In order to arrive at the expression (2.11) we have used the property (2.7) and the equations of motion

$$
D_{+} D_{-} \Phi^{\mu}+\Gamma_{\rho \sigma}^{-\mu} D_{+} \Phi^{\rho} D_{-} \Phi^{\sigma}=0,
$$

which follow from the action (2.1). If we want the algebra (2.11) to be identical to the algebra (2.3) we have to require the following properties of $J_{ \pm}$

$$
J_{ \pm \nu}^{\mu} J_{ \pm \mu}^{\rho}=-\delta_{\nu}^{\rho},
$$




$$
\mathcal{N}_{\mu \nu}^{\rho}\left(J_{ \pm}\right)=0
$$

Thus the supersymmetry algebra requires that $J_{ \pm}$correspond to two complex structures.

This is the full description of the most general $\mathrm{N}=(2,2)$ sigma model. Thus the target manifold should be a bihermitian complex manifold (i.e., there are two complex structures and a metric is Hermitian with respect to both) and the two complex structures should be covariantly constant, with respect to the different connections however. The main question we would like to address is under what conditions on two complex structures (or their Kähler forms) one can construct the appropriate affine connections such that (2.7) is fulfilled.

Before answering this question let us explore the properties of the target manifold. The vanishing of the Nijenhuis tensor (2.13) and the condition (2.7) imply that the complex structures should preserve the torsion

$$
H_{\delta \nu \lambda}=J_{ \pm \delta}^{\sigma} J_{ \pm \nu}^{\rho} H_{\sigma \rho \lambda}+J_{ \pm \lambda}^{\sigma} J_{ \pm \delta}^{\rho} H_{\sigma \rho \nu}+J_{ \pm \nu}^{\sigma} J_{ \pm \lambda}^{\rho} H_{\sigma \rho \delta}
$$

Introducing the Kähler forms

$$
\omega_{ \pm} \equiv g J_{ \pm}
$$

and using equations $(2.7)$ one can find $d \omega_{ \pm}$

$$
\left(d \omega_{ \pm}\right)_{\rho \mu \nu}= \pm\left(H_{\sigma \rho \mu} J_{ \pm \nu}^{\sigma}+H_{\sigma \mu \nu} J_{ \pm \rho}^{\sigma}+H_{\sigma \nu \rho} J_{ \pm \mu}^{\sigma}\right)
$$

where we use the following normalization to the exterior differential

$$
\left(d \omega_{ \pm}\right)_{\lambda \sigma \gamma}=\frac{1}{2}\left(\partial_{\lambda} \omega_{ \pm \sigma \gamma}+\partial_{\sigma} \omega_{ \pm \gamma \lambda}+\partial_{\gamma} \omega_{ \pm \lambda \sigma}\right) .
$$

Now by combining (2.17) and (2.19) one arrives at the following property

$$
H_{\mu \nu \rho}=-J_{+\mu}^{\lambda} J_{+\nu}^{\sigma} J_{+\rho}^{\gamma}\left(d \omega_{+}\right)_{\lambda \sigma \gamma}=J_{-\mu}^{\lambda} J_{-\nu}^{\sigma} J_{-\rho}^{\gamma}\left(d \omega_{-}\right)_{\lambda \sigma \gamma} .
$$

The first equality in expression (2.21) can be considered as a definition of the torsion. Starting from the complex structure, let say $J_{+}$, one can construct the torsion (2.21) such that $\nabla_{\rho}^{(+)} J_{+\nu}^{\mu}=0$ where $\nabla^{(+)}$is defined in (2.7), [23]. Thus the only non-trivial constraint which arises from $(2.21)$ is the relation between $\left(J_{+}, \omega_{+}\right)$and $\left(J_{-}, \omega_{-}\right)$. However, in the present form the relation (2.21) tells us very little about the geometry of the target manifold.

To summarize the discussion, let us state the geometric data which gives the necessary and sufficient conditions for the target manifold to admit the $\mathrm{N}=(2,2)$ sigma model. The target manifold $\mathcal{M}\left(g, J_{ \pm}\right)$should be equipped with two complex structures $J_{ \pm}$and the metric $g$ which is Hermitian with respect to both complex structures $J_{ \pm}$. In addition, the complex structures $J_{ \pm}$together with their Kähler forms $\omega_{ \pm}$(2.18) should satisfy the relation (2.21), defining the torsion $H$. 
There is another property which arises in the construction, the closedness of torsion form, $d H=0$. However this property is only related to the fact that the equations of motion are derived from the Lagrangian, which may involve the closed torsion only. At the level of symmetries and classical dynamics, this property is not required (i.e., the field equations and the $\mathrm{N}=2$ supersymmetry remain consistent even when $d H \neq 0$ in the model) and therefore in our further discussion we treat the closedness of the torsion as a secondary property.

\section{Poisson structures of $(2,2)$ sigma model}

In this section we reformulate some of the previous conditions in new terms. In particular we give a new form to the constraint (2.21) relating two complex structures involved in the second supersymmetry transformation, so that it reveals the Poisson structures on the target manifold of the $\mathrm{N}=(2,2)$ sigma model.

It turns out that it is appropriate to describe the target manifold geometry in terms of contravariant antisymmetric tensor fields instead of covariant ones like metric $g$, exterior forms $\omega_{ \pm}$(2.18) and their differentials, as it has been done in previous section. Before doing this, let us remind the relevant notions related to the differential calculus of contravariant tensor fields.

Given two antisymmetric contravariant tensor fields (multivectors) $\alpha$ and $\beta$ of the rank $|\alpha|$ and $|\beta|$ respectively one may define a Schouten bracket [12] of them being a tensor of $\operatorname{rank}|\alpha|+|\beta|-1$ :

$$
\begin{gathered}
\alpha=\alpha^{i_{1} \ldots i_{|\alpha|}} \partial_{i_{1}} \wedge \ldots \wedge \partial_{i_{|\alpha|}}, \beta=\beta^{i_{1} \ldots i_{|\beta|}} \partial_{i_{1}} \wedge \ldots \wedge \partial i_{|\beta|} \\
{[\alpha, \beta]_{s} \equiv\left(|\beta| \partial_{j} \alpha^{i_{1} \ldots i_{|\alpha|}} \beta^{j i_{|\alpha|+1} \ldots i_{|\beta|+|\alpha|-1}}-|\alpha| \alpha^{i_{1} \ldots i_{|\alpha|-1} j} \partial_{j} \beta^{i_{|\alpha|} \ldots i_{|\beta|+|\alpha|-1}}\right) \partial_{i_{1}} \wedge \ldots \wedge \partial_{i_{|\alpha|+|\beta|-1}}}
\end{gathered}
$$

The Schouten bracket can be thought of as an extension of the Lie bracket of the vector fields to the case of multivectors. Being defined for inhomogeneous tensor fields (by linearity), this bracket can be identified to the Batalin-Vilkovisky antibracket [13] on the odd cotangent bundle $\Pi T^{*} \mathcal{M}$ of the target manifold $\mathcal{M}$. The bracket (3.22) has properties of the odd Poisson bracket (or anti-bracket that is the same) which is well-known in physics from the Batalin-Vilkovisky field-antifield formalism: (graded) symmetry, (graded) Jacobi identity and (graded) Liebnitz rule:

$$
\begin{aligned}
{[\alpha, \beta]_{s} } & =-(-1)^{(|\alpha|-1)(|\beta|-1)}[\beta, \alpha]_{s} \\
{\left[\alpha,[\beta, \gamma]_{s}\right]_{s} } & =\left[[\alpha, \beta]_{s}, \gamma\right]_{s}+(-1)^{(|\alpha|-1)(|\beta|-1)}\left[\beta,[\alpha, \gamma]_{s}\right]_{s} \\
{[\alpha, \beta \wedge \gamma]_{s} } & =[\alpha, \beta]_{s} \wedge \gamma+(-1)^{(|\alpha|-1)|\beta|} \beta \wedge[\alpha, \gamma]_{s}
\end{aligned}
$$


Notice that the Schouten bracket of two even rank tensors is symmetric, so the Schouten nilpotency condition is not automatically satisfied for an even rank tensor. If second rank tensor $p$ is Schouten-nilpotent on $\mathcal{M}$,

$$
p=p^{\mu \nu} \partial_{\mu} \wedge \partial_{\nu}, \quad[p, p]_{s}=0
$$

it defines the conventional (even) Poisson bracket

$$
\{f, g\} \equiv p(d f, d g)=p^{\mu \nu} \partial_{\mu} f \partial_{\nu} g, \quad f(x), g(x) \in C^{\infty}(\mathcal{M}),
$$

which is a bilinear map $C^{\infty}(\mathcal{M}) \times C^{\infty}(\mathcal{M}) \rightarrow C^{\infty}(\mathcal{M})$. Because of (3.26) the Poisson bracket (3.27) has ordinary antisymmetry property and satisfies standard Leibnitz rule and Jacobi identity.

The tensor $p$ satisfying (3.26) is called a Poisson tensor and the manifold which admits such $p$ is called a Poisson manifold (for a review of the Poisson geometry see, e.g. [24]). Notice that the Poisson geometry does not imply that $p^{\mu \nu}$ has an inverse, moreover the rank of $p$ may be varying from point to point on the manifold. In the case of nondegenerate Poisson tensor we have a symplectic manifold.

Two Poisson tensors $p_{1}$ and $p_{2}$ are called compatible [25] if they Schouten commute (i.e., $\left.\left[p_{1}, p_{2}\right]_{s}=0\right)$. Apparently any linear combination of two compatible Poisson tensors $p_{a}, a=1,2$ is a Poisson tensor again. So if the manifold manifold is equipped with two compatible Poisson structures there exists a continuos family of the Poisson brackets $p(k)=$ $k^{a} p_{a}, k=\left(k^{1}, k^{2}\right) \in R P^{1}$ with any two elements $p(k), p(l), \forall k, l \in R P^{1}$ compatible to each other. The compatibility property was first studied for Poisson structures in relation to the integrable systems [25].

Now let us reformulate the constraint (2.21) in the new terms. Introduce the contravariant tensors $j_{ \pm}$which are dual to two-forms $\omega_{ \pm}(2.18)$

$$
j_{+}^{\mu \nu} \omega_{+\nu \rho}=\delta_{\rho}^{\mu}, \quad j_{-}^{\mu \nu} \omega_{-\nu \rho}=\delta_{\rho}^{\mu}
$$

Using the metric, we raise the indices in (2.21) and rewrite this condition in the equivalent form in terms of $j_{ \pm}$:

$$
2 h=-\left[j_{+}, j_{+}\right]_{s}=\left[j_{-}, j_{-}\right]_{s}
$$

where

$$
h^{\mu \nu \rho}=g^{\mu \lambda} g^{\nu \sigma} g^{\rho \gamma} H_{\lambda \sigma \gamma} .
$$

Just in terms of $j_{ \pm}$the condition (3.29) has the form

$$
\left[j_{+}, j_{+}\right]_{s}+\left[j_{-}, j_{-}\right]_{s}=0
$$


Thus for the complex structures $J_{ \pm}$on the bihermitian manifold, the condition (3.29) implies that there exists an affine connection such that the conditions (2.7) are satisfied. Using the fact that both connections respect the metric (i.e., $\nabla_{\rho}^{( \pm)} g_{\mu \nu}=0$ ), one can show that $(2.7$ ) implies $j_{-}$should Schouten commute to $j_{+}$

$$
\left[j_{-}, j_{+}\right]_{s}=0
$$

Introducing the linear combinations of $j_{ \pm}$

$$
l=\frac{1}{2}\left(j_{+}+j_{-}\right), \quad m=\frac{1}{2}\left(j_{+}-j_{-}\right)
$$

the algebra (3.29) and (3.32) can be equivalently rewritten as follows

$$
[l, l]_{s}=0, \quad[m, m]_{s}=0, \quad[l, m]_{s}=-h .
$$

Thus $l$ and $m$ are Schouten-nilpotent ${ }^{3}$. Notice that the relations (3.34) are consistent because of the Schouten bracket properties (3.25) only, without requiring the torsion three-form $H$ entering 3 -vector $h$ to be closed.

As a result of the previous discussion we see that $l$ and $m$ are Poisson structures which are not compatible however. Therefore the resulting manifold is a bi-Poisson (i.e., there are two Poisson structures, not an infinite family of the Poisson tensors). Let us now summarize this result. The manifold with two complex structures $J_{+}, J_{-}$and with the metric $g$ which is hermitian with respect to both structures will admit the on-shell algebra (2.3), (2.10) and (2.12) if and only if $\left(J_{+} \pm J_{-}\right) g^{-1}$ are Poisson structures. We have already proved this statement in one direction: starting from the bihermitian manifold and using the specific form of the covariant constancy (2.7) of the complex structures, we have shown that the algebra (3.34) is satisfied. In opposite direction, having the bihermitian manifold with the algebra $[l, l]_{s}=0$ and $[m, m]_{s}=0$ one immediately arrives at the property (3.31) which is equivalent to the property (2.21) which defines the torsion such that (2.7) is satisfied. Therefore the onshell algebra ${ }^{4}(2.3),(2.10)$ and (2.12) is fulfilled. In order to have a Lagrangian formulation of the model one has to require that $d H=0$ which in fact leads to the extra requirement on the relation between the Poisson structures $l$ and $m$. However it does not affect their existence or non existence.

To summarize the discussion, the target manifold $\mathcal{M}$ of the $\mathrm{N}=(2,2)$ sigma model can be completely characterized by the following geometric data: $\mathcal{M}$ should be equipped with two

\footnotetext{
${ }^{3}$ Unlike $j_{ \pm}, l$ and $m$ may be degenerate in a generic situation, for the details see the next section.

${ }^{4}$ On-shell algebra means that we are allowed to use the equations of motion while calculating the algebra. The problem of existence of an off-shell realization of the same algebra is a different problem which is not discussed here.
} 
complex structures $J_{ \pm}$and the metric $g$ which is Hermitian with respect to both complex structures. In addition $\left(J_{+}+J_{-}\right) g^{-1}$ and $\left(J_{+}-J_{-}\right) g^{-1}$ should be the Poisson bi-vectors. The torsion $H$ is defined through the Schouten bracket of these two Poisson structures.

We would like to stress the fact that when either $l$ or $m$ vanishes identically on $\mathcal{M}$ we end up with the standard Kähler case and the well-known realization of $\mathrm{N}=2$ supersymmetry algebra. Therefore the Kähler case is automatically included in the above discussion and corresponds to the "singular" case when either $J_{+}=J_{-}$or $J_{+}=-J_{-}$.

\section{Poisson geometry of the target manifold: local analysis and special cases}

In this section we would like to analyse the immediate consequences of the fact that the manifold is bi-Poisson. We explore the local geometry of this manifold. In particular we study the structure and intersections of the symplectic leaves of two Poisson structures defined by bi-vectors $l$ and $m$.

Also we discuss in more details some special cases of this geometry and propose a program for the classification of this type of geometries.

We start by recalling the picture where one can (locally) think of a Poisson manifold as a union of symplectic leaves fitting together in a smooth way. Let $\mathcal{M}$ be a Poisson manifold with the Poisson structure $p^{\mu \nu}, \mu, \nu=1,2, \ldots, d$ and let us choose the point $x_{0}$ such that in its neighbourhood $\operatorname{rank}(p)$ is constant ${ }^{5}$. Contraction of the bi-vector $p$ to any one-form $e$, being a section of $T^{*} \mathcal{M}, \quad e \notin \operatorname{ker}(p)$, provides a vector field which is called a locally Hamiltonian, if locally $e=d f$ for some function $f \in C^{\infty}(\mathcal{M})$. So we have a set of locally Hamiltonian vector fields

$$
v_{k}^{\mu}=p^{\mu \nu} \partial_{\nu} f_{k}, \quad k=1,2, \ldots, n, n=d-\operatorname{rank}\left(p\left(x_{0}\right)\right)
$$

which are in involution, i.e. the Lie bracket of two locally Hamiltonian vector fields is a locally Hamiltonian field again

$$
\left\{v_{i}, v_{j}\right\}_{\text {Lie }}^{\mu}=p^{\mu \rho} \partial_{\rho}\left(\left(\partial_{\nu} f_{j}\right) p^{\nu \lambda}\left(\partial_{\lambda} f_{i}\right)\right)
$$

Thus due to the Frobenius theorem, locally they generate an integral submanifold $S$ through a point $x_{0}$ and it is always possible to introduce the local coordinates $x^{\mu}=\left(x^{A}, x^{i}\right), A=$ $1, \ldots, d-\operatorname{rank}(p), i=d-\operatorname{rank}(p)+1, \ldots, d$ in the neighbourhood of $x_{0}$ such that $S$ can

\footnotetext{
${ }^{5}$ Such a point is called a regular. On a non-regular Poisson manifold there are singular points which do not have this property [24]. We do not discuss here these points and their neighbourhoods.
} 
be described by $x^{i}=$ const and $x^{A}$ are the coordinates on $S$. The restriction of a Poisson bracket to the functions on a submanifold $S$ yields again a Poisson bracket. Thus $\left.p\right|_{S}$ is a non degenerate Poisson structure on $S$, i.e. a symplectic structure. As a result in these coordinates $x^{\mu}=\left(x^{A}, x^{i}\right), p$ has the following form

$$
p^{\mu \nu}=\left(\begin{array}{ll}
p^{A B} & 0 \\
0 & 0
\end{array}\right)
$$

If one wishes by doing an appropriate coordinate transformations in $x^{A}$ one can bring $\left.p\right|_{S}$ to the canonical Darboux form. Thus one can conclude that the Poisson manifold is foliated by the symplectic leaves. In a generic coordinate system there is locally complete set of independent Casimir functions $\left\{f_{a}(x)\right\}$ of $p$ which have vanishing Poisson bracket with any function from $C^{\infty}(\mathcal{M})$. Then in this coordinates locally the symplectic leaf can be given by the conditions $f_{a}(x)=$ conts.

Now let us apply this picture to our model with two Poisson tensors, $l$ and $m$. Since the combinations $(l \pm m)$ are nondegenerate, the Poisson brackets, defined by the bi-vectors $l$ and $m$, can not have common Casimir functions. Thus one can choose the following two sets of Casimir functions: $\left\{\phi_{a}(x), a=1, \ldots, n_{l}\right\}$ which span the kernel of $l$ and $\left\{\psi_{i}(x), i=1, \ldots, n_{m}\right\}$ which span the kernel of $m$. The conditions $\phi_{a}(x)=$ const define the symplectic submanifolds $\mathcal{S}_{l}$ of dimension ${ }^{6}\left(d-n_{l}\right)$ which are symplectic leaves of the Poisson structure $l$. The conditions $\psi_{i}(x)=$ const define the symplectic submanifolds $\mathcal{S}_{m}$ of dimension $\left(d-n_{m}\right)$ which are symplectic leaves of the Poisson structure $m$. Moreover the conditions $\phi_{a}(x)=$ const and $\psi_{i}(x)=$ const together define the submanifolds $\mathcal{S}_{l m}$ of the whole manifold $\mathcal{M}$

$$
\mathcal{S}_{l m}=\mathcal{S}_{l} \bigcap \mathcal{S}_{m} \subset \mathcal{M}
$$

and $\mathcal{S}_{l m}$ is a submanifold of two symplectic manifolds, $\mathcal{S}_{l}$ and $\mathcal{S}_{m}$. Therefore the submanifolds $\mathcal{S}_{l m}$ can be described in terms of symplectic geometry. There are three numbers which would characterize $\mathcal{S}_{l m}$ : the dimension $\mathcal{S}_{l m}$, the rank of restriction of the symplectic structure to $\mathcal{S}_{l m}$ from $\mathcal{S}_{l}$ and the rank of restriction of the symplectic structure to $\mathcal{S}_{l m}$ from $\mathcal{S}_{m}$. Thus these three numbers together with the dimensions of the symplectic leaves $\mathcal{S}_{l}$ and $\mathcal{S}_{m}$ can be used for the description of the local geometry of the given type of the manifolds. The Kähler geometry is the extreme case of this geometry when, for example, the symplectic leaves $\mathcal{S}_{l}$ coincide with the whole manifold $\mathcal{M}$ and $\mathcal{S}_{m}$ vanish.

The kernels of $l$ and $m$ are related in an obvious way to the the (left) kernels of $\left(J_{+}+J_{-}\right)$ and $\left(J_{-}-J_{+}\right)$. In the work [17] it has been proved that the spaces orthogonal to the kernels of

\footnotetext{
${ }^{6}$ Since the manifold $\mathcal{M}$ is even dimensional complex manifold the codimension of symplectic leaf is even and thus the number of Casimir functions is also even.
} 
$\left(J_{+} \pm J_{-}\right)$and $\left[J_{+}, J_{-}\right] \equiv J_{+} J_{-}-J_{-} J_{+}$(the product of $J_{ \pm}$is understood here as a standard matrix multiplication) are always integrable, and this proof was enough complicated. In the context of the Poisson geometry these facts become trivial as one can see from above discussion. The spaces orthogonal to the kernels of $\left(J_{+} \pm J_{-}\right)$correspond to the symplectic leaves of $l$ and $m$ respectively. The space, which is orthogonal to the kernel of $\left[J_{+}, J_{-}\right]$, corresponds to $\mathcal{S}_{l m}$.

To clarify the picture presented one can look at a special cases (extreme in a sense) of this geometry. Let us start form the case when the complex structures commute $\left(\left[J_{+}, J_{-}\right]=0\right)$.

This case has been studied in the details in the original work [4]. The corresponding geometry is given by the local product of two symplectic manifolds. The symplectic leaf of $l(m)$ is the kernel of $m(l)$. The product of two complex structures gives rise to an integrable local product structure (i.e., $\Pi=J_{+} J_{-}, \Pi^{2}=I$ and $\mathcal{N}_{\mu \nu}^{\rho}(\Pi)=0$ ). Hence the submanifolds projected by $1 / 2(I \pm \Pi)$ are symplectic leaves.

Another interesting example corresponds to the case when $\left[J_{+}, J_{-}\right]$nondegenerate on $\mathcal{M}$. Thus $l$ and $m$ are invertiable and we can define their inverses

$$
\mathbf{l}_{\mu \rho} l^{\rho \nu}=\delta_{\nu}^{\mu}, \quad \mathbf{m}_{\mu \rho} m^{\rho \nu}=\delta_{\nu}^{\mu} .
$$

The 2-forms $\mathbf{l}$ and $\mathbf{m}$ are closed

$$
d \mathbf{l}=0, \quad d \mathbf{m}=0
$$

and the manifold $\mathcal{M}$ is bi-symplectic. In this case the torsion is expressible through the Nijenhuis tensor of $J_{ \pm}$

$$
\mathcal{N}_{\nu \rho}^{\mu}\left(J_{+}, J_{-}\right)=2\left[J_{+}, J_{-}\right]_{\sigma}^{\mu} g^{\sigma \gamma} H_{\gamma \nu \rho}
$$

where $\left[J_{+}, J_{-}\right]$is invertiable. Thus the torsion vanishes if and only if both complex structures are simultaneously integrable. In the case of zero torsion we have the bi-Kähler case and the Poisson structures $l$ and $m$ are compatible (i.e., $[l, m]_{s}=0$ ). In this case we have a continuous family of Poisson structures given by $p(t)=\left(t^{1} l+t^{2} m\right)$ (where $t=\left(t^{1}, t^{2}\right) \in R P^{1}$ is any element of a real projective space) and smoothly interpolating between $l$ and $m$.

In this section we have discussed mostly the geometrical problems related to the structure of symplectic leaves of the two Poisson brackets. A separate consideration is required for the geometry around the irregular points where the Poisson tensor(s) change their rank.

\section{$5 \quad \mathrm{~N}=(4,4)$ supersymmetric sigma model}

In this section we discuss the general supersymetric sigma model with $N>2$. As was established in [4] there are interesting models with $\mathrm{N}=4$. Below we show that these $\mathrm{N}=(4,4)$ 
sigma models have a rich underlying Poisson geometry.

The dimensional analysis requires the following form of the additional supersymmetry transformations

$$
\delta^{(i)}(\epsilon) \Phi^{\mu}=\epsilon^{+} D_{+} \Phi^{\nu} J_{+\nu}^{(i) \mu}(\Phi)+\epsilon^{-} D_{-} \Phi^{\nu} J_{-\nu}^{(i) \mu}(\Phi), \quad i=2,3, \ldots, N
$$

We want the transformations (5.42) to be the symmetries of the action (2.1) and satisfy the on-shell algebra

$$
\left[\delta^{i}\left(\epsilon_{1}\right), \delta^{j}\left(\epsilon_{2}\right)\right] \Phi^{\mu}=2 i \delta^{i j} \epsilon_{1}^{+} \epsilon_{2}^{+} \partial_{+} \Phi^{\mu}+2 i \delta^{i j} \epsilon_{1}^{-} \epsilon_{2}^{-} \partial_{=} \Phi^{\mu}
$$

where $\delta^{1}(\epsilon)$ is the manifest supersymmetry transformation given by (2.2).

The analysis goes along the lines presented in Section 2. The additional supersymmetry requires $J_{ \pm}^{(i)}$ to be the complex structures. $J_{ \pm}^{(i)}$ satisfy the conditions $(2.6),(2.7)$ and (2.16), while (2.15) and (2.17) are replaced by the more stringent conditions

$$
\begin{gathered}
J_{ \pm \nu}^{(i) \mu} J_{ \pm \rho}^{(j) \nu}+J_{ \pm \nu}^{(j) \mu} J_{ \pm \rho}^{(i) \nu}=-2 \delta^{i j} \delta_{\rho}^{\mu} \\
H_{\delta \nu \lambda} \delta^{i j}=J_{ \pm \delta}^{(i) \sigma} J_{ \pm \nu}^{(j) \rho} H_{\sigma \rho \lambda}+J_{ \pm \lambda}^{(i) \sigma} J_{ \pm \delta}^{(j) \rho} H_{\sigma \rho \nu}+J_{ \pm \nu}^{(i) \sigma} J_{ \pm \lambda}^{(j) \rho} H_{\sigma \rho \delta}
\end{gathered}
$$

Thus for $\mathrm{N}=4$ supersymmetry ${ }^{7}$ the manifold has two quaternionic structures and therefore a dimensionality that is a multiple of four. Introducing the Kähler form $\omega_{ \pm}^{(i)}=g J_{ \pm}^{(i)}$ the relations (2.19) and (2.21) are still valid. This description of $\mathrm{N}=(4,4)$ models has been presented in [4] (see also [26] and [27] for the recent discussion of $\mathrm{N}=4$ models).

Following the discussion of Section 3 we can rewrite the above conditions in terms of the contravariant antisymmetric tensors $j_{ \pm}^{(i)}=-J_{ \pm}^{(i)} g^{-1}$. After the simple calculation we arrive at the algebra

$$
\left[j_{+}^{(i)}, j_{+}^{(j)}\right]_{s}=-2 \delta^{i j} h, \quad\left[j_{-}^{(i)}, j_{-}^{(j)}\right]_{s}=2 \delta^{i j} h, \quad\left[j_{+}^{(i)}, j_{-}^{(j)}\right]_{s}=0
$$

As before we can introduce the linear combinations of $j_{ \pm}^{(i)}$

$$
l^{(i)}=\frac{1}{2}\left(j_{+}^{(i)}+j_{-}^{(i)}\right), \quad m^{(i)}=\frac{1}{2}\left(j_{+}^{(i)}-j_{-}^{(i)}\right)
$$

and rewrite the algebra (5.47) as follows

$$
\left[l^{(i)}, l^{(j)}\right]_{s}=0, \quad\left[m^{(i)}, m^{(j)}\right]_{s}=0, \quad\left[l^{(i)}, m^{(j)}\right]_{s}=-\delta^{i j} h .
$$

Thus there are six Poisson structures $l^{(i)}$ and $m^{(i)}$ such that $l^{(i)}\left(m^{(i)}\right)$ is compatible with all other Poisson structures except $m^{(i)}\left(l^{(i)}\right)$. As in Section 3 the algebra (5.48) gives the

\footnotetext{
${ }^{7} \mathrm{~N}=3$ supersymmetry implies $\mathrm{N}=4$ and there is a bound on the number of supersymmetries, $N \leq 4$ (for the details see, e.g. [2]).
} 
necessary and sufficient conditions for bi-quaternionic manifolds (the metric should be hermitian with respect to all complex structures) to admit the on-shell $\mathrm{N}=(4,4)$ supersymmetry algebra (5.43).

The case when either $l^{(i)}$ or $m^{(i)}$ are zeros (i.e., either $J_{+}^{(i)}=-J_{-}^{(i)}$ or $J_{+}^{(i)}=J_{-}^{(i)}$ ) corresponds to the hyperKähler geometry. The hyperKähler geometry corresponds to $\mathrm{N}=(4,4)$ supersymmetric sigma models without torsion, $\mathrm{H}=0$. This case is automatically included in the previous discussion.

\section{Discussions}

In this short paper we argue that the appearance of the Poisson structures is a common feature of two dimensional sigma models with extended supersymmetry of type $(p, q)$ with $2 \leq p$ and $2 \leq q$. We gave the explicit description of the most general models with $(2,2)$ and $(4,4)$ supersymmetries. We briefly discussed the underlying geometry of the models. However, our discussion of the geometry is sketchy and we plan to come back to a detailed description of this type of geometry elsewhere.

We believe that the new description of the general $\mathrm{N}=2$ sigma model in terms of Poisson geometry opens new perspectives on old problems. Specifically we hope to study the twisted versions of the presented general $\mathrm{N}=2$ sigma models. Another problem where the new description may be useful is the general description of $\mathrm{N}=2$ boundary conditions (D-branes) of the corresponding sigma model [28].

Acknowledgements: We are grateful to Ulf Lindström for useful discussions and for reading and commenting on the manuscript. SLL is thankful to Lars Brink and Robert Marnelius for their very warm hospitality at Chalmers University where he has begun this work and to Seif Randjbar-Daemi at ICTP where the work was mostly completed. The work of SLL was supported in parts by the STINT fellowship, INTAS grant 00-262, the RFBR grant 00-02-17956 and the grant E-00-33-184 from Russian Ministry of Education.

\section{A Appendix: $(1,1)$ supersymmetry}

In the appendix we list $\mathrm{N}=1$ supersymmetric conventions we use mostly following [5].

We use $(+,=)$ as worldsheet indices, and $(+,-)$ as two-dimensional spinor indices. We also use superspace conventions where the pair of spinor coordinates of the two-dimensional superspace are labelled $\theta^{ \pm}$, and the covariant derivatives $D_{ \pm}$and supersymmetry generators 
$Q_{ \pm}$satisfy

$$
D_{+}^{2}=i \partial_{+}, \quad D_{-}^{2}=i \partial_{=}, \quad\left\{D_{+}, D_{-}\right\}=0, \quad Q_{ \pm}=i D_{ \pm}+2 \theta^{ \pm} \partial_{\underline{\underline{H}}}
$$

where $\partial_{\underline{\underline{H}}}=\partial_{0} \pm \partial_{1}$. In terms of the covariant derivatives, a supersymmetry transformation of a superfield $\Phi$ is then given by

$$
\delta \Phi \equiv i\left(\varepsilon^{+} Q_{+}+\varepsilon^{-} Q_{-}\right) \Phi=-\left(\varepsilon^{+} D_{+}+\varepsilon^{-} D_{-}\right) \Phi+2 i\left(\varepsilon^{+} \theta^{+} \partial_{+}+\varepsilon^{-} \theta^{-} \partial_{=}\right) \Phi
$$

\section{References}

[1] B. Zumino, "Supersymmetry And Kahler Manifolds," Phys. Lett. B 87 (1979) 203.

[2] L. Alvarez-Gaumé and D. Z. Freedman, "Geometrical Structure And Ultraviolet Finiteness In The Supersymmetric Sigma Model," Commun. Math. Phys. 80 (1981) 443.

[3] S. J. Gates, "Superspace Formulation Of New Nonlinear Sigma Models," Nucl. Phys. B 238 (1984) 349.

[4] S. J. Gates, C. M. Hull and M. Roček, "Twisted Multiplets And New Supersymmetric Nonlinear Sigma Models," Nucl. Phys. B 248 (1984) 157.

[5] N. J. Hitchin, A. Karlhede, U. Lindström and M. Roček, "Hyperkahler Metrics And Supersymmetry," Commun. Math. Phys. 108 (1987) 535.

[6] N. Ikeda, "Two-dimensional gravity and nonlinear gauge theory," Annals Phys. 235 (1994) 435 [arXiv:hep-th/9312059].

[7] P. Schaller and T. Strobl, "Poisson structure induced (topological) field theories," Mod. Phys. Lett. A 9 (1994) 3129 [arXiv:hep-th/9405110].

[8] A. S. Cattaneo and G. Felder, "A path integral approach to the Kontsevich quantization formula," Commun. Math. Phys. 212 (2000) 591 [arXiv:math.qa/9902090].

[9] M. Kontsevich, "Deformation quantization of Poisson manifolds, I," arXiv:q$\operatorname{alg} / 9709040$.

[10] A. S. Cattaneo, G. Felder and L. Tomassini, "From local to global deformation quantization of Poisson manifolds," arXiv:math.qa/0012228.

[11] B. V. Fedosov, "A Simple Geometrical Construction of Deformation Quantization," J. Diff. Geom. 40 (1994) 213. 
[12] J. A. Schouten, "On differential operators of first order in tensor calculus," in Convengo Int. Diff. Geom. Italia, 1953 Ed. Cremonese, Roma, 1954.

[13] I. A. Batalin and G. A. Vilkovisky, "Gauge Algebra And Quantization," Phys. Lett. B 102 (1981) 27.

[14] T. Buscher, U. Lindström and M. Roček, "New Supersymmetric Sigma Models With Wess-Zumino Terms," Phys. Lett. B 202 (1988) 94.

[15] M. Roček, "Modified Calabi-Yau manifolds with torsion," IASSNS-HEP-91-43 Submitted to Proc. of Mirror Symmetry Workshop, MSRI, Berkeley, CA, May 1991

[16] M. Roček, K. Schoutens and A. Sevrin, "Off-shell WZW models in extended superspace," Phys. Lett. B 265 (1991) 303.

[17] I. T. Ivanov, B. b. Kim and M. Roček, "Complex Structures, Duality And WZW Models In Extended Superspace," Phys. Lett. B 343 (1995) 133 [arXiv:hep-th/9406063].

[18] A. Sevrin and J. Troost, "Off-shell formulation of $N=2$ non-linear sigma-models," Nucl. Phys. B 492 (1997) 623 [arXiv:hep-th/9610102].

[19] J. Bogaerts, A. Sevrin, S. van der Loo and S. Van Gils, "Properties of semi-chiral superfields," Nucl. Phys. B 562 (1999) 277 [arXiv:hep-th/9905141].

[20] P. Spindel, A. Sevrin, W. Troost and A. Van Proeyen, "Complex Structures On Parallelized Group Manifolds And Supersymmetric Sigma Models," Phys. Lett. B 206 (1988) 71.

[21] P. Spindel, A. Sevrin, W. Troost and A. Van Proeyen, "Extended Supersymmetric Sigma Models On Group Manifolds. 1. The Complex Structures," Nucl. Phys. B 308 (1988) 662 .

[22] A. Sevrin, W. Troost, A. Van Proeyen and P. Spindel, "Extended Supersymmetric Sigma Models On Group Manifolds. 2. Current Algebras," Nucl. Phys. B 311 (1988) 465 .

[23] K. Yano, "Differential geometry on complex and almost complex spaces" (Pergamon, Oxford, 1965)

[24] I. Vaisman, "Lectures on the Geometry of Poisson Manifolds", Progress in Mathematics, Vol 118 (Birkhäuser, Basel, 1994)

[25] F. Magri, "A Simple Model Of The Integrable Hamiltonian Equation," J. Math. Phys. 19 (1978) 1156. 
[26] S. J. Gates, "Why are there so many N=4 superstrings?," Phys. Lett. B 338 (1994) 31 [arXiv:hep-th/9410149].

[27] S. J. Gates and S. V. Ketov, "2D $(4,4) 1$ hypermultiplets. I: Diversity for N $=4$ models," Phys. Lett. B 418 (1998) 111 [arXiv:hep-th/9504077].

[28] U. Lindström and M. Zabzine, " $\mathrm{N}=2$ Boundary conditions for non-linear sigma models and Landau-Ginzburg models," arXiv:hep-th/0209098. 